\title{
Association between nontraditional lipid profiles and peripheral arterial disease in Chinese adults with hypertension
}

Congcong Ding ${ }^{1 \dagger}$, Yang Chen ${ }^{1 \dagger}$, Yumeng Shi ${ }^{1}$, Minghui $\mathrm{Li}^{1}$, Lihua Hu${ }^{1}$, Wei Zhou ${ }^{1,2}$, Tao Wang ${ }^{1,2}$, Lingjuan Zhu ${ }^{1,2}$, Xiao Huang ${ }^{1}$, Huihui Bao ${ }^{1,2^{*}}$ and Xiaoshu Cheng ${ }^{1,2^{*}}$ (D)

\begin{abstract}
Background: Data on the relationship between nontraditional lipid profiles [total cholesterol (TC)/high-density lipoprotein cholesterol (HDL-C) ratio, triglyceride (TG)/HDL-C ratio, low-density lipoprotein cholesterol (LDL-C)/HDL$\mathrm{C}$ ratio, non-high-density lipoprotein cholesterol (non-HDL-C)] and the risk of peripheral artery disease (PAD) are limited. The present study investigated the relationship of nontraditional lipid indices with PAD in hypertensive patients.
\end{abstract}

Methods: This cross-sectional study was performed among 10,900 adults with hypertension. Participants were diagnosed with PAD when their ankle-brachial index (ABI) was $<0.9$. The association between nontraditional lipid profiles and PAD was examined using multivariate logistic regression analysis and the restricted cubic spline.

Results: All nontraditional lipid indices were independently and positively associated with PAD in a dose-response fashion. After multivariable adjustment, the per SD increments of the TC/HDL-C, TG/HDL-C, LDL-C/HDL-C ratios and non-HDL-C were all significantly associated with $37,14,40$, and $24 \%$ higher risk for PAD, respectively. The adjusted ORs $(95 \% \mathrm{Cl})$ for PAD were $1.77(1.31,2.40), 1.71(1.25,2.34), 2.03(1.50,2.74)$, and $1.70(1.25,2.31)$ when comparing the highest tertile to the lowest tertile of the TC/HDL-C, TG/HDL-C, LDL-C/HDL-C ratios and non-HDL-C, respectively.

Conclusions: Among Chinese hypertensive adults, all nontraditional lipid indices were positively associated with $P A D$, and the LDL-C/HDL-C and TC/HDL-C ratios were better than the other nontraditional lipid indices for predicting PAD. These findings may improve the risk stratification of cardiovascular disease and dyslipidemia management.

Trial registration: CHiCTR, ChiCTR1800017274. Registered 20 July 2018.

Keywords: Nontraditional lipid profiles, Peripheral artery disease, Hypertension, Ankle-brachial index, Cholesterol, Chinese adults, Epidemiology

\footnotetext{
*Correspondence: huihui_bao77@126.com; xiaoshumenfan126@163.com

${ }^{+}$Congcong Ding and Yang Chen contributed equally and should be considered as co-first authors

'Department of Cardiovascular Medicine, the Second Affiliated Hospital of

Nanchang University, No. 1 Minde Road, Nanchang 330006, Jiangxi Province,

China

Full list of author information is available at the end of the article
}

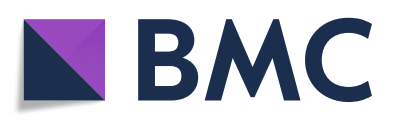

(- The Author(s). 2020 Open Access This article is licensed under a Creative Commons Attribution 4.0 International License, which permits use, sharing, adaptation, distribution and reproduction in any medium or format, as long as you give appropriate credit to the original author(s) and the source, provide a link to the Creative Commons licence, and indicate if changes were made. The images or other third party material in this article are included in the article's Creative Commons licence, unless indicated otherwise in a credit line to the material. If material is not included in the article's Creative Commons licence and your intended use is not permitted by statutory regulation or exceeds the permitted use, you will need to obtain permission directly from the copyright holder. To view a copy of this licence, visit http://creativecommons.org/licenses/by/4.0/. The Creative Commons Public Domain Dedication waiver (http://creativecommons.org/publicdomain/zero/1.0/) applies to the data made available in this article, unless otherwise stated in a credit line to the data. 


\section{Background}

Peripheral artery disease (PAD) is a clinical manifestation of systemic atherosclerosis. PAD is estimated to affect 200 million people globally, and it is a strong predictor of cardiovascular causes of death [1]. However, PAD is not fully understood and treated [2, 3]. The ankle-brachial index (ABI), which is commonly known as the ratio of the systolic blood pressure (SBP) measured at the ankle and arm, is a reliable, inexpensive, and noninvasive test for the diagnosis of PAD [4]. However, more than half of patients with PAD are asymptomatic, and an absence of routine $\mathrm{ABI}$ measurement in the primary care setting exists in most countries. Agents that reliably indicate subclinical atherosclerosis for patients who are at high risk of suffering from PAD are urgently needed.

Traditional lipid parameters, such as abnormal rates of high-density lipoprotein cholesterol (HDL-C) and lowdensity lipoprotein cholesterol (LDL-C), are common risk factors for PAD [5]. Notably, an increasing number of studies have indicated the development and progression of atherosclerosis under conditions of abnormal nontraditional lipid profiles. The triglyceride (TG)/HDL$\mathrm{C}$ ratio may be a favourable predictor of cardiovascular disorders [6-9], and many studies have suggested that the total cholesterol (TC)/HDL-C ratio is also closely related to cardiovascular disease (CVD) [10-12]. Growing evidence has validated the relationship between the LDL-C/HDL-C ratio and the carotid plaque score [13], carotid intima media thickness [14], and PAD [12]. In addition, non-high-density lipoprotein cholesterol (nonHDL-C), which is the sum of cholesterol in lipoproteins other than HDL-C, was also shown to be a better predictor for major adverse cardiovascular events than LDL-C in a prospective observation study [15].

Increasing research shows a strong link between hypertension and PAD [16]. Therefore, the identification of lipid-related risk factors in people with hypertension will help improve strategies for population-based screening and prevention in subjects who are at risk for vascular complications. However, few studies have investigated the association between all nontraditional lipid indices and PAD risk. No previous study has examined this association in a population with hypertension. The present study comprehensively determined the association between nontraditional lipid profiles and PAD and identified the best surrogate indicators for predicting the risk of PAD risk in Chinese adults with hypertension.

\section{Methods}

\section{Subject population and design}

The subject population in this study was selected from the ongoing China Hypertension Registry Study (registration number: ChiCTR1800017274), a large-scale, real- world observational registry study. It was designed to determine the prevalence and control of hypertension and evaluate the prognostic factors of hypertension from this registry of hypertension in rural areas of southern China. All study subjects were hypertension patients who were at least 18 years old. Eligible participants had at least one of the following factors: (1) individuals with an SBP > $140 \mathrm{mmHg}$ or/and diastolic blood pressure (DBP) $\geq 90$ $\mathrm{mmHg}$ in the relaxed sitting position at the initial screening; (2) individuals receiving antihypertensive medications; or (3) individuals with a history of hypertension. Participants with neurological abnormalities or who were unable to complete the follow-up visit according to the research protocol were excluded from the study. The ethics committee of the Institute of Biomedicine, Anhui Medical University, approved the study. All study subjects signed a written informed consent before their enrolment in this study.

Ultimately, the present study recruited 14,268 eligible study subjects in Wuyuan County in Jiangxi Province, China from March 2018 to August 2018. After excluding subjects without hypertension $(n=34)$ and subjects with missing ABI $(n=3328)$ and lipid profile values $(n=6)$, a total of 10,900 hypertensive patients were included in the final analyses (Fig. 1).

\section{Data collection}

The research staff were strictly trained to perform health interviews for the study population. A questionnaire was used to collect demographic information including age, sex, lifestyle data (e.g., alcohol drinking status and smoking status), medical history and medication information. The anthropometric measurement indicators included weight and height. Blood pressure (BP) was obtained using an electronic sphygmomanometer (Omron; Dalian, China) after the individual rested in a seated position for more than $5 \mathrm{~min}$. Three measurements on the right arm were performed with one-minute intervals between successive readings, and the mean value was calculated.

After more than $12 \mathrm{~h}$ of overnight fasting, all venous blood samples of the study participants were collected, frozen and transported to Biaojia Biotechnology in Shenzhen in Guangdong Province, China. Automatic clinical analysers (Beckman Coulter, USA) were used to measure serum fasting glucose, lipids (HDL-C, LDL-C, TG and $\mathrm{TC}$ ), creatinine and total homocysteine (tHcy). All laboratory operations complied with the standardization and certification procedures.

The TC/HDL-C, TG/HDL-C, and LDL-C/HDL-C ratios were calculated as TC, TG, and LDL-C divided by HDL$\mathrm{C}$, respectively. Non-HDL-C was calculated as HDL-C subtracted from TC. The body mass index (BMI) was calculated by dividing the weight by the height squared. The estimated glomerular filtration rate (eGFR) was calculated 


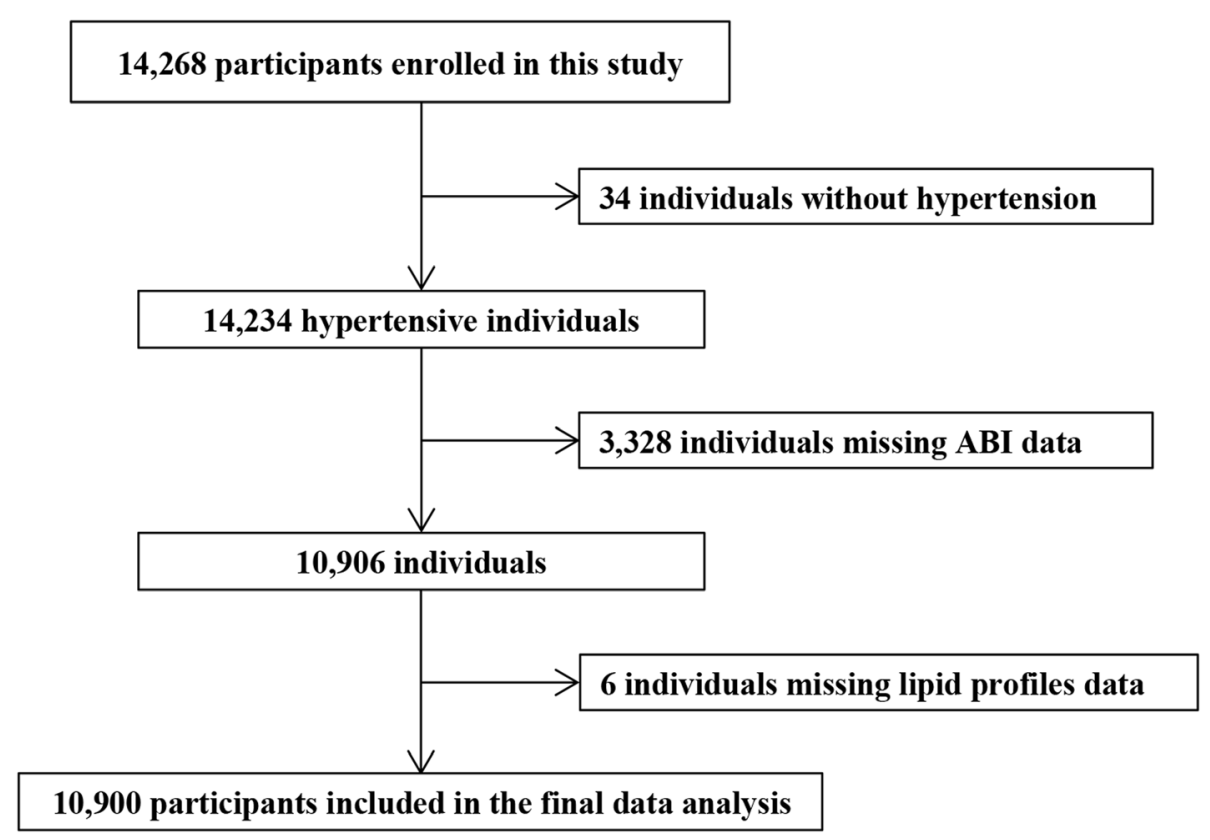

Fig. 1 Flow chart of participants

using the formula derived from the Chronic Kidney Disease Epidemiology Collaboration [17].

\section{Pad}

Participants were diagnosed with PAD when an $\mathrm{ABI}<$ 0.9 existed in either leg [18]. According to standard clinical guidelines, the ABI was automatically measured with the individuals lying flat on their back [19]. Measurements were performed after the participants had rested for at least $10 \mathrm{~min}$ using the BP-203RPE III device (Omron, Kyoto, Japan). The ABI was calculated as the highest SBP at the ankles divided by the highest SBP of the right or left upper arms. The lower ABI values calculated for the left and right ankles were used in the analysis.

\section{Covariables}

The covariates were selected based on their clinical importance, statistical significance in the univariable analysis of their associations with PAD, and the potential confounder effect estimates individually changing by at least $10 \%$. The covariates were age, sex, BMI, SBP, DBP, smoking status, alcohol drinking status, fasting glucose, tHcy, eGFR, self-reported diabetes, self-reported stroke, use of lipoprotein-lowering drugs, and use of antihypertensive drugs.

\section{Statistical analyses}

The mean $\pm \mathrm{SD}$ or the median (25th percentile, 75 th percentile) and number (\%) were used to describe continuous data and categorical data, respectively.
Differences in data characteristics with or without PAD were compared using Student's t-test for normally distributed data, nonparametric Mann-Whitney test for abnormally distributed data, and a chi-squared test for categorical data. Logistic regression analyses were performed to assess the association between nontraditional lipid indices (the non-HDL-C, LDL-C/HDL-C, TG/ HDL-C and TC/HDL-C ratios) and PAD by presenting the odds ratio (OR) and 95\% confidence interval (CI) with adjustment for pertinent variables. Nontraditional lipid profiles were assessed by establishing models for both continuous data per SD increment and categorical data using tertiles with the lowest tertile (T1) as the reference group. Trend tests were calculated using the nontraditional lipid profile tertile categories as continuous data. Restricted cubic spline (smooth fitting curve) was performed to visually evaluate the association with nontraditional lipid profiles of PAD. Potential effect modifiers were evaluated by subgroup and interaction analyses.

All the data analyses were performed using $R$ version 3.4.3 (www.R-project.org) and EmpowerStates (www. empowerstats.com). A 2 -sided $P<0.05$ was considered statistically significant.

\section{Results}

Baseline characteristics

The final data analyses included 10,900 patients with hypertension. Table 1 presents the participant characteristics for all subjects and subjects with and without PAD. Overall, the mean age was 63.9 years and $47.1 \%$ of 
Table 1 Characteristics of study participants with or without PAD

\begin{tabular}{|c|c|c|c|c|}
\hline Variables & Total $(n=10,900)$ & non-PAD $(n=10,590)$ & $\operatorname{PAD}(n=310)$ & $P$ value \\
\hline Age, years & $63.9 \pm 9.3$ & $63.6 \pm 9.1$ & $71.6 \pm 10.0$ & $<0.001$ \\
\hline Male, n (\%) & $5129(47.1 \%)$ & $4958(46.8 \%)$ & $171(55.2 \%)$ & 0.004 \\
\hline $\mathrm{BMl}, \mathrm{kg} / \mathrm{m}^{2}$ & $23.6 \pm 3.8$ & $23.6 \pm 3.8$ & $22.5 \pm 3.9$ & $<0.001$ \\
\hline $\mathrm{SBP}, \mathrm{mm} \mathrm{Hg}$ & $148.5 \pm 18.2$ & $148.4 \pm 18.0$ & $152.8 \pm 22.7$ & $<0.001$ \\
\hline $\mathrm{DBP}, \mathrm{mm} \mathrm{Hg}$ & $89.1 \pm 11.5$ & $89.2 \pm 11.5$ & $84.1 \pm 11.5$ & $<0.001$ \\
\hline Current smoking, n (\%) & $2868(26.3 \%)$ & 2745 (25.9\%) & $123(39.7 \%)$ & $<0.001$ \\
\hline Current alcohol drinking, n (\%) & $2469(22.7 \%)$ & 2409 (22.8\%) & $60(19.4 \%)$ & 0.159 \\
\hline Self-reported diabetes, n (\%) & $1176(10.8 \%)$ & $1145(10.8 \%)$ & $31(10.0 \%)$ & 0.650 \\
\hline Self-reported stroke, n (\%) & $708(6.5 \%)$ & $667(6.3 \%)$ & $41(13.2 \%)$ & $<0.001$ \\
\hline \multicolumn{5}{|l|}{ Laboratory results } \\
\hline Fasting glucose, $\mathrm{mmol} / \mathrm{L}$ & $6.2 \pm 1.6$ & $6.2 \pm 1.6$ & $6.1 \pm 1.5$ & 0.439 \\
\hline Total homocysteine, $\mu \mathrm{mol} / \mathrm{L}$ & $18.0 \pm 11.0$ & $17.9 \pm 10.8$ & $22.5 \pm 17.1$ & $<0.001$ \\
\hline $\mathrm{eGFR}, \mathrm{mL} \cdot \mathrm{min}^{-1} \cdot 1.73 \mathrm{~m}^{-2}$ & $88.7 \pm 20.4$ & $89.1 \pm 20.1$ & $74.8 \pm 23.3$ & $<0.001$ \\
\hline $\mathrm{TC}, \mathrm{mmol} / \mathrm{L}$ & $5.1 \pm 1.1$ & $5.1 \pm 1.1$ & $5.1 \pm 1.2$ & 0.966 \\
\hline $\mathrm{TG}, \mathrm{mmol} / \mathrm{L}$ & $1.4(1.0-2.1)$ & $1.4(1.0-2.1)$ & $1.4(1.0-2.1)$ & 0.069 \\
\hline LDL-C, mmol/L & $3.0 \pm 0.8$ & $3.0 \pm 0.8$ & $3.1 \pm 0.9$ & 0.156 \\
\hline $\mathrm{HDL}-\mathrm{C}, \mathrm{mmol} / \mathrm{L}$ & $1.6 \pm 0.4$ & $1.6 \pm 0.4$ & $1.5 \pm 0.4$ & 0.001 \\
\hline $\mathrm{TC} / \mathrm{HDL}-\mathrm{C}$ ratio & $3.4 \pm 0.8$ & $3.4 \pm 0.8$ & $3.5 \pm 0.9$ & $<0.001$ \\
\hline TG/HDL-C ratio & $0.9(0.6-1.5)$ & $0.9(0.6-1.5)$ & $0.9(0.6-1.5)$ & 0.304 \\
\hline LDL-C/HDL-C ratio & $2.0 \pm 0.7$ & $2.0 \pm 0.6$ & $2.1 \pm 0.7$ & $<0.001$ \\
\hline Non-HDL-C, mmol/L & $3.6 \pm 1.0$ & $3.6 \pm 1.0$ & $3.6 \pm 1.1$ & 0.163 \\
\hline \multicolumn{5}{|l|}{ Medication use, $\mathrm{n}(\%)$} \\
\hline Antihypertensive drugs & $7155(65.6 \%)$ & $6930(65.4 \%)$ & $225(72.6 \%)$ & 0.009 \\
\hline Glucose-lowering drugs & $572(5.2 \%)$ & $554(5.2 \%)$ & $18(5.8 \%)$ & 0.654 \\
\hline Lipoprotein-lowering drugs & $381(3.5 \%)$ & $369(3.5 \%)$ & $12(3.9 \%)$ & 0.715 \\
\hline Antiplatelet drugs & $429(3.9 \%)$ & 409 (3.9\%) & $20(6.5 \%)$ & 0.021 \\
\hline
\end{tabular}

Data are expressed as mean \pm standard deviation or median (interquartile range) and numbers (percentage) as appropriate Abbreviations: PAD Peripheral arterial disease; BMI Body mass index; SBP Systolic blood pressure; DBP Diastolic blood pressure; eGFR Estimated glomerular filtration rate; TC Total cholesterol; TG Triglyceride; HDL-C High-density lipoprotein cholesterol; LDL-C Low-density lipoprotein cholesterol; non-HDL-C Non-high-density lipoprotein cholesterol

participants were males. Compared with the participants without PAD, those with PAD tended to have a higher age, SBP, tHcy, TC/HDL-C ratio, and LDL-C/HDL-C ratio; be male and a current smoker; have a higher rate of self-reported stroke and antihypertensive or antiplatelet drug use; and have a lower BMI, DBP, eGFR and HDL$C$ level (all $P<0.05$ ). No significant differences were found between participants with or without PAD in terms of fasting glucose, LDL-C, TG, TC, TG/HDL-C ratio, non-HDL-C level, current alcohol drinking, selfreported diabetes, or use of glucose-lowering drugs or lipoprotein-lowering drugs.

\section{Nontraditional lipid profiles and PAD}

The association between nontraditional lipid profiles and PAD was assessed using multiple logistic regression analyses, and the results are listed in Table 2. Only the continuous $\mathrm{TC} / \mathrm{HDL}-\mathrm{C}$ and $\mathrm{LDL}-\mathrm{C} / \mathrm{HDL}-\mathrm{C}$ ratios were significantly and positively associated with $\mathrm{PAD}$ in the crude model. However, after multivariable adjustment, the per SD increment of the TC/HDL-C ratio, TG/ HDL-C ratio, LDL-C/HDL-C ratio, and non-HDL-C level were all significantly associated with a $37,14,40$, and $24 \%$ higher risk for PAD, respectively. Consistently, when the nontraditional lipid profiles were assessed as tertiles, the adjusted ORs (95\% CI) were 1.77 (1.31, 2.40), 1.71 (1.25, 2.34), $2.03(1.50,2.74)$, and 1.70 (1.25, 2.31) for the top tertiles of the TC/HDL-C, TG/HDL-C, LDL-C/HDL-C ratios, and non-HDL-C, respectively, compared with the lowest tertiles. Additionally, the association between the four nontraditional lipid profiles and PAD was likely to be linear (all $P$ for trend $<0.001$ ). Similar trends were found when nontraditional lipid profiles were assessed as quintiles (see Additional file 1: 
Table 2 Odds ratio of PAD according to continuous or tertiles of nontraditional lipid profiles

\begin{tabular}{|c|c|c|c|c|c|c|}
\hline \multirow[t]{2}{*}{ Variables } & \multirow[t]{2}{*}{$\mathbf{N}$} & \multirow{2}{*}{$\begin{array}{l}\text { Events, } \\
\mathrm{n}(\%)\end{array}$} & \multicolumn{2}{|l|}{ Crude } & \multicolumn{2}{|l|}{ Model 1} \\
\hline & & & OR $(95 \% \mathrm{Cl})$ & $P$ value & OR $(95 \% \mathrm{Cl})$ & $P$ value \\
\hline TC/HDL-C ratio (Per 1 SD increase) & 10,900 & $310(2.8 \%)$ & $1.20(1.08,1.34)$ & $<0.001$ & $1.37(1.22,1.54)$ & $<0.001$ \\
\hline \multicolumn{7}{|l|}{ Tertiles of $\mathrm{TC} / \mathrm{HDL}-\mathrm{C}$ ratio } \\
\hline $\mathrm{T} 1(<3.0)$ & 3633 & $90(2.5 \%)$ & 1.00 (reference) & & 1.00 (reference) & \\
\hline $\mathrm{T} 2(3.0-3.7)$ & 3633 & $103(2.8 \%)$ & $1.15(0.86,1.53)$ & 0.343 & $1.36(1.01,1.84)$ & 0.045 \\
\hline $\mathrm{T} 3(\geq 3.7)$ & 3634 & $117(3.2 \%)$ & $1.31(0.99,1.73)$ & 0.058 & $1.77(1.31,2.40)$ & $<0.001$ \\
\hline$P$ for trend & & & & 0.057 & & $<0.001$ \\
\hline TG/HDL-C ratio (Per 1 SD increase) & 10,900 & $310(2.8 \%)$ & $0.93(0.82,1.06)$ & 0.302 & $1.14(1.01,1.29)$ & 0.029 \\
\hline \multicolumn{7}{|l|}{ Tertiles of TG/HDL-C ratio } \\
\hline $\mathrm{T} 1(<0.7)$ & 3633 & $100(2.8 \%)$ & 1.00 (reference) & & 1.00 (reference) & \\
\hline $\mathrm{T} 2(0.7-1.3)$ & 3633 & $106(2.9 \%)$ & $1.06(0.80,1.40)$ & 0.672 & $1.27(0.95,1.70)$ & 0.111 \\
\hline $\mathrm{T} 3(\geq 1.3)$ & 3634 & $104(2.9 \%)$ & $1.04(0.79,1.38)$ & 0.778 & $1.71(1.25,2.34)$ & $<0.001$ \\
\hline$P$ for trend & & & & 0.779 & & $<0.001$ \\
\hline LDL/HDL-C ratio (Per 1 SD increase) & 10,900 & $310(2.8 \%)$ & $1.22(1.10,1.36)$ & $<0.001$ & $1.40(1.25,1.57)$ & $<0.001$ \\
\hline \multicolumn{7}{|l|}{ Tertiles of LDL/HDL-C ratio } \\
\hline $\mathrm{T} 1(<1.7)$ & 3633 & $91(2.5 \%)$ & 1.00 (reference) & & 1.00 (reference) & \\
\hline $\mathrm{T} 2(1.7-2.2)$ & 3632 & $91(2.5 \%)$ & $1.00(0.75,1.34)$ & 0.999 & $1.23(0.91,1.68)$ & 0.182 \\
\hline $\mathrm{T} 3(\geq 2.2)$ & 3635 & $128(3.5 \%)$ & $1.42(1.08,1.87)$ & 0.012 & $2.03(1.50,2.74)$ & $<0.001$ \\
\hline$P$ for trend & & & & 0.009 & & $<0.001$ \\
\hline Non-HDL-C (Per 1 SD increase) & 10,900 & $310(2.8 \%)$ & $1.08(0.97,1.21)$ & 0.163 & $1.24(1.10,1.39)$ & $<0.001$ \\
\hline \multicolumn{7}{|l|}{ Tertiles of Non-HDL-C } \\
\hline $\mathrm{T} 1(<3.1)$ & 3621 & $90(2.5 \%)$ & 1.00 (reference) & & 1.00 (reference) & \\
\hline $\mathrm{T} 2(3.1-3.9)$ & 3633 & $112(3.1 \%)$ & $1.25(0.94,1.65)$ & 0.123 & $1.58(1.17,2.12)$ & 0.003 \\
\hline $\mathrm{T} 3(\geq 3.9)$ & 3646 & $108(3.0 \%)$ & $1.20(0.90,1.59)$ & 0.213 & $1.70(1.25,2.31)$ & $<0.001$ \\
\hline$P$ for trend & & & & 0.223 & & $<0.001$ \\
\hline
\end{tabular}

Model 1 was adjusted for age, sex, BMI, SBP, DBP, smoking status, alcohol drinking status, fasting glucose, total homocysteine, eGFR, self-reported diabetes, selfreported stroke, lipoprotein-lowering drugs, and antihypertensive drugs

Abbreviations: PAD Peripheral arterial disease; OR Odd ratio; 95\% Cl 95\% Confidence interval; TC Total cholesterol; TG Triglyceride; HDL-C High-density lipoprotein cholesterol; LDL-C Low-density lipoprotein cholesterol; non-HDL-C Non-high-density lipoprotein cholesterol

Table S1). Further analyses using restricted cubic spline confirmed the linearly positive association between the four nontraditional lipid profiles and the risk of PAD (Fig. 2).

\section{Subgroup analyses}

Subgroup analyses by stratification of the major covariates were performed to further confirm that the findings were reliable in the presence of potential confounders. None of the stratified variables, including sex, age, BMI, smoking, drinking, SBP, tHcy and fasting glucose, significantly modified the association between the four types of nontraditional lipid profiles and the risk of PAD (all $P$-interaction $>0.05$ ) (see Additional file 1: Fig. S1-4).

\section{Discussion}

In this sample of large rural residents, the nontraditional lipid profiles were all positively related to the risk of PAD in hypertension participants in China. The restricted cubic spline indicated that the relationship of the four nontraditional lipid indices with PAD was linear. Moreover, the findings suggested that the TC/HDL$\mathrm{C}$ and LDL-C/HDL-C ratios were better early predictors of PAD risk than other nontraditional lipid indices.

To date, the relationship between nontraditional lipid indices and arteriosclerotic cardiovascular disease (ASCVD) has not been comprehensively examined. Lee et al. [11] performed a prospective study of 107 participants with type 2 diabetes in Taiwan and found that $\mathrm{TC} / \mathrm{HDL}-\mathrm{C}$ showed an inverse trend in changing in the ABI $(\beta=-0.212,95 \% \mathrm{CI}:-0.043--0.001)$. Another post hoc data analysis from a randomized controlled trial of 1599 community-based elderly participants reported that the TC/HDL-C ratio (per SD increment, OR: 1.31; 95\% CI: 1.14-1.49), non-HDL-C level (OR: 1.15; 95\% CI: 1.01-1.31) and LDL-C/HDL-C ratio (OR: 1.25 ; 95\% CI: 1.10-1.43) were significantly correlated with PAD [12]. Moreover, another population-based study of the elderly 

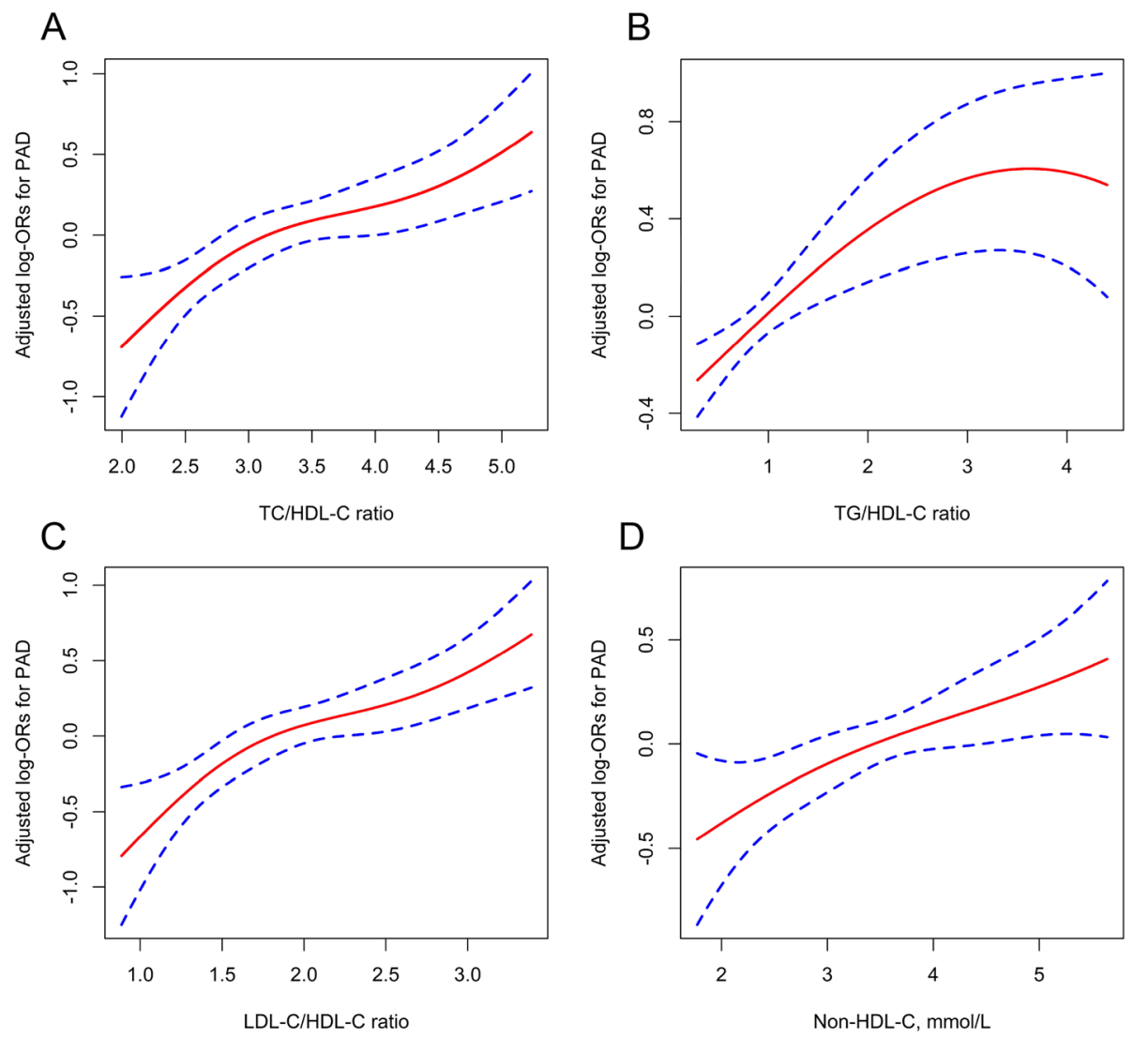

Fig. 2 The association between the TC/HDL-C (a), TG/HDL-C (b), and LDL-C/HDL-C ratios (c) and the non-HDL-C (d) level and the risk of PAD. The solid line and dashed line represent the estimated values and their corresponding $95 \%$ confidence interval, respectively. The adjustment factors included age, sex, BMI, SBP, DBP, smoking status, alcohol drinking statuse, fasting glucose, total homocysteine, eGFR, self-reported diabetes, selfreported stroke, lipoprotein-lowering drugs, and antihypertensive drugs

in rural China also observed a significant association between the LDL-C/HDL-C ratio and PAD (highest compared with the lowest tertile; OR: 2.56 ; $95 \% \mathrm{CI}, 1.37$ to 4.81) [20]. Additionally, Ridker et al. [21] and Pradhan et al. [22] separately reported the results concerning the association between the TC/HDL-C ratio and PAD in male physicians and female health professionals in the United States. Both studies yielded the same results that the TC/HDL-C ratio was significantly associated with PAD. However, Tongdee et al. found that the TC/HDLC, TG/HDL-C and LDL-C/HDL-C ratios failed to predict early subclinical atherosclerosis in perimenopausal/ menopausal women [23]. A cross-sectional survey [24] based on 2982 elderly Beijing residents found that the TG/HDL-C ratio was not associated with a low ABI $(\mathrm{ABI} \leq 0.9)$ (highest compared with the lowest quartile; OR: $1.64 ; 95 \%$ CI, 0.88 to $3.07 ; P \geq 0.05)$. This study also showed that the trends of the association between the $\mathrm{TC} / \mathrm{HDL}-\mathrm{C}$ ratio and the risk of PAD were nonlinear. The PAD risk was almost identical on the left side of inflexion and then increased linearly when the TC/HDL-C ratio $\geq 3$. However, no studies have assessed the association between nontraditional lipid profiles and PAD in hypertensive populations.

Previous studies did not find that the linear relationship, to some extent, may be attributed to differences in patient characteristics, tHcy levels, or adjustment of confounders. First, the participants in the present study had hypertension, and the study by Lee et al. included only participants with type 2 diabetes. Hypertension may exhibit heterogeneity, and different diseases have different injury saturation point. Second, the lipid levels are significantly different based on region and diet, and the baseline lipid levels may impact the levels of lipid ratios and the prevalence of PAD. The high-carbohydrate diets of Chinese populations may contribute to hypertriglyceridemia, and the standard Western high-fat diets are more likely to induce hypercholesterolemia [25]. The present study was performed in a population with highcarbohydrate diets, and the studies of Ridker et al. and Pradhan et al. were undertaken in regions with the standard high-fat diets. Indeed, obvious geographical differences in PAD incidence also exist in China [26]. The present study was performed in the inland areas of the 
south, but the studies of Liang et al. and Zhan et al. were conducted in northern areas, while the study by Chi et al. was performed in the coastal areas. Third, the mean baseline tHcy was $18.0 \mu \mathrm{mol} / \mathrm{L}$ in the present study, much higher than normal levels. High tHcy could causes disorders of lipid metabolism and further atherosclerotic disease $[27,28]$. Therefore, the association between nontraditional lipid profiles and PAD in a group of low tHcy concentrations cannot be examined. Overall, the current studies are just hypothesis-generating, and further investigations are necessary to consolidate the results of this study.

The exact mechanisms by which the nontraditional lipid indices could predict the risk of PAD were unclear, but using nontraditional lipid indices to predict PAD is biologically plausible. First, significant alterations in nontraditional lipid profiles, which are likely the result of dyslipidaemia in the early stages of PAD, may be characterized as a state of miniaturized low-density lipoprotein particle (LDL-P), and decreased high-density lipoprotein particle (HDL-P). The TC/HDL-C ratio, as a manifestation of atherogenic particle load, was associated with LDL-P, and the particle differences in low-density lipoprotein (LDL) may be attributed to a source of residual risk of cardiovascular events and a unique lipoprotein signature for PAD [29, 30]. In addition, previous studies showed that an elevated serum TG/HDL-C ratio independently predicted a decrease in LDL-P size [31]. Previous studies showed that small dense LDL (sdLDL) promoted pro-atherogenic modifications with the characteristics of incremental flux into the arterial intima, prolonged circulation time and reduced LDL receptor affinity [32, 33]. Moreover, the TC/HDL-C and TG/ HDL-C ratios may reflect a decrease in mature large-size HDL-P, which is related to anti-atherosclerosis $[25,34$, 35]. Non-HDL-C is a recognized risk factor for ASCVD because of its containment all of the atherogenic lipoproteins, and an epidemiological survey showed that non-HDL-C was a stronger lipid parameter of atherogenesis than LDL-C [15]. Overall, the TC/HDL-C, TG/ HDL-C and non-HDL-C ratios indirectly suggest early PAD risk by reflecting the size and density of LDL-P, HDL-P and other atherosclerotic factors, such as chylomicron, very-low density lipoprotein (VLDL), and intermediate density lipoprotein (IDL), which are all closely related to CVD. Second, the TC/HDL-C and TG/HDL$\mathrm{C}$ ratios were strongly related to insulin resistance, whereas insulin resistance was closely related to the development of PAD [36-38]. Third, most participants (98.7\%) in the present study had hyperhomocysteinaemia (HHcy, defined as tHcy $\geq 10 \mu \mathrm{mol} / \mathrm{L}$ ) simultaneously, and HHcy may reflect the clinical significance of nontraditional lipid indices by mediating the changes in blood lipid levels and increased adverse effects of lipids on atherosclerosis. In addition to reducing the apolipoprotein A-I composite, HHcy also reduces the high-density lipoprotein (HDL) content in circulation via the promotion of HDL-C clearance [28, 39]. Furthermore, elevated LDL levels in patients with HHcy could be more likely to produce atherosclerotic disease [40, 41]. Therefore, the LDL-C/HDL-C ratio may be a good indicator for identifying the risk of early atherosclerosis, and it can replace the standard lipid profile in the HHcy population. Because a lot of studies have come to the same conclusion that nontraditional lipid profiles would better represent the potential atherosclerotic evolution, using nontraditional lipid profiles to assess the risk of atherosclerotic diseases is reasonable.

\section{Study strengths and limitations}

This study is currently the largest study to assess the association between nontraditional lipid profiles and the risk of PAD in hypertension patients. Nevertheless, several potential limitations of this study are noteworthy. First, this study was a cross-sectional study, which makes it difficult to explain the causal relationship between non-traditional lipid profiles and PAD. Second, the study population comprised rural hypertensive patients in southern China, and the study subjects were over 18 years old. Therefore, these results cannot be generalized to other age groups, regions, or types of diseases.

\section{Conclusion}

Among Chinese hypertensive adults, all of the nontraditional lipid indices (the TC/HDL-C, TG/HDL-C, LDL$\mathrm{C} / \mathrm{HDL}-\mathrm{C}$ ratios, and non-HDL-C) were independently and positively associated with PAD. In addition, the TC/ HDL-C and LDL-C/HDL-C ratios better predicted the risk of PAD than other nontraditional lipid indices. Therefore, the use of these nontraditional lipid profiles, which are inexpensive and easy to calculate in clinical practice, may improve the risk stratification of ASCVD and select favourable candidates for aggressive lipoprotein-lowering therapy.

\section{Supplementary Information}

Supplementary information accompanies this paper at https://doi.org/10. 1186/s12944-020-01407-3.

\footnotetext{
Additional file 1: Table S1. Odds ratio of PAD according to continuous or quintiles of nontraditional lipid profiles. Fig. S1. The association between the TC/HDL-C ratio (per SD increment) and the risk of peripheral arterial disease (PAD) in various subgroups*. . Adjusted, if not stratified, for age, sex, BMI, SBP, DBP, smoking status, alcohol drinking status, fasting glucose, total homocysteine, eGFR, self-reported diabetes, self-reported stroke, lipoprotein-lowering drugs, and antihypertensive drugs. History of diabetes was defined as self-reported diabetes, or use of glucose-lowering drugs. Fig. S2. The association between the TG/HDL-C ratio (per SD increment) and the risk of peripheral arterial disease (PAD) in various subgroups*. Adjusted, if not stratified, for age, sex, BMI, SBP,
} 
DBP, smoking status, alcohol drinking status, fasting glucose, total homocysteine, eGFR, self-reported diabetes, self-reported stroke, lipoproteinlowering drugs, and antihypertensive drugs. History of diabetes was defined as self-reported diabetes, or use of glucose-lowering drugs. Fig. S3 The association between the LDL-C/HDL-C ratio (per SD increment) and the risk of peripheral arterial disease (PAD) in various subgroups. . $\mathrm{Ad}$ justed, if not stratified, for age, sex, BMI, SBP, DBP, smoking status, alcohol drinking status, fasting glucose, total homocysteine, eGFR, self-reported diabetes, self-reported stroke, lipoprotein-lowering drugs, and antihypertensive drugs. History of diabetes was defined as self-reported diabetes, or use of glucose-lowering drugs. Fig. S4. The association between the non-HDL-C (per SD increment) and the risk of peripheral arterial disease (PAD) in various subgroups.. *Adjusted, if not stratified, for age, sex, BMI, SBP, DBP, smoking status, alcohol drinking status, fasting glucose, total homocysteine, eGFR, self-reported diabetes, self-reported stroke, lipoprotein-lowering drugs, and antihypertensive drugs. History of diabetes was defined as self-reported diabetes, or use of glucose-lowering drugs.

\section{Abbreviations}

PAD: Peripheral arterial disease; TC: Total cholesterol; TG: Triglyceride; HDLC: High-density lipoprotein cholesterol; LDL-C: Low-density lipoprotein cholesterol; non-HDL-C: Non-High-density lipoprotein cholesterol; ABI: Anklebrachial index; SBP: Systolic blood pressure; DBP: Diastolic blood pressure; CVD: Cardiovascular disease; tHcy: Total homocysteine; BMI: Body mass index; eGFR: Estimated glomerular filtration rate; OR: Odds ratio; Cl: Confidence interval; ASCVD: Arteriosclerotic cardiovascular disease; LDL-P: Low density lipoprotein particle; HDL-P: High density lipoprotein particle; sdLDL: Small dense LDL; VLDL: Very low density lipoprotein; IDL: Intermediate density lipoprotein; HHcy: Hyperhomocysteinaemia

\section{Acknowledgements}

The authors acknowledge the contribution of the all staff who participated in this study as well as the study participants.

\section{Authors' contributions}

CCD and $Y C$ participated in the literature search, data analysis, and data interpretation. CCD wrote the manuscript. LHH extracted and collected data. $X H, Y M S, M H L, L H H, W Z$, TW, and LJZ conceived of the study and participated in its design and coordination. HHB and XSC participated in the study design and provided critical revision. All authors read and approved the final manuscript.

\section{Funding}

This work was supported by the Science and Technology Innovation Platform Project of Jiangxi Province (Grant number: 20165BCD41005).

\section{Availability of data and materials}

The datasets generated and analysed during the current study are not publicly available because this study is still on-going and the follow-up is not finished, but they are available from the corresponding author on reasonable request.

\section{Ethics approval and consent to participate}

The study was conducted in accordance with the Declaration of Helsinki and was approved by the Ethics Committee of the Anhui Medical University Biomedical Institute. Informed written consent was obtained from all patients before their enrolment in this study.

\section{Consent for publication}

All the authors have consented to the publication of this study.

\section{Competing interests}

The authors declare that they have no competing interests.

\section{Author details}

'Department of Cardiovascular Medicine, the Second Affiliated Hospital of Nanchang University, No. 1 Minde Road, Nanchang 330006, Jiangxi Province, China. ${ }^{2}$ Center for Prevention and Treatment of Cardiovascular Diseases, the
Second Affiliated Hospital of Nanchang University, Nanchang, Jiangxi Province, China.

Received: 7 August 2020 Accepted: 19 October 2020

Published online: 03 November 2020

\section{References}

1. Fowkes FGR, Aboyans V, Fowkes FJl, McDermott MM, Sampson UKA, Criqui $\mathrm{MH}$. Peripheral artery disease: epidemiology and global perspectives. Nat Rev Cardiol. 2017;14:156-70.

2. Hess CN, Norgren L, Ansel GM, Capell WH, Fletcher JP, Fowkes F, et al. A structured review of antithrombotic therapy in peripheral artery disease with a focus on revascularization: a TASC (InterSociety consensus for the Management of Peripheral Artery Disease) initiative. Circulation. 2017;135: 2534-55.

3. Campia U, Gerhard-Herman M, Piazza G, Goldhaber SZ. Peripheral artery disease: past, present, and future. Am J Med. 2019;132:1133-41.

4. Aboyans V, Criqui MH, Abraham P, Allison MA, Creager MA, Diehm C, et al. Measurement and interpretation of the ankle-brachial index. Circulation. 2012;126:2890-909.

5. Aponte J. The prevalence of asymptomatic and symptomatic peripheral arterial disease and peripheral arterial disease risk factors in the US population. Holist Nurs Pract. 2011;25:147-61.

6. Kim JE, Yu M, Kim YC, Min S, Ha J, Lee JP, et al. Ratio of triglyceride to highdensity lipoprotein cholesterol and risk of major cardiovascular events in kidney transplant recipients. Clin Exp Nephrol. 2019;23:1407-17.

7. Sultani R, Tong DC, Peverelle M, Lee YS, Baradi A, Wilson AM. Elevated triglycerides to high-density lipoprotein cholesterol (TG/HDL-C) ratio predicts Long-term mortality in high-risk patients. Heart Lung Circ. 2020;29: 414-21.

8. Zhan X, Yang M, Zhou R, Wei X, Chen Y, Chen Q. Triglyceride to highdensity lipoprotein cholesterol ratio is associated with increased mortality in older patients on peritoneal dialysis. Lipids Health Dis. 2019;18.

9. Su Y, Zhang R, Xu R, Wang H, Geng H, Pan M, et al. Triglyceride to highdensity lipoprotein cholesterol ratio as a risk factor of repeat revascularization among patients with acute coronary syndrome after firsttime percutaneous coronary intervention. J Thorac Dis. 2019:11:5087-95.

10. Arsenault BJ, Rana JS, Stroes ES, Despres JP, Shah PK, Kastelein JJ, et al. Beyond low-density lipoprotein cholesterol: respective contributions of nonhigh-density lipoprotein cholesterol levels, triglycerides, and the total cholesterol/high-density lipoprotein cholesterol ratio to coronary heart disease risk in apparently healthy men and women. J Am Coll Cardiol. 2009; 55:35-41.

11. Lee I, Huang C, Lee W, Lee H, Sheu WH. High total-to-HDL cholesterol ratio predicting deterioration of ankle brachial index in Asian type 2 diabetic subjects. Diabetes Res Clin Pract. 2008;79:419-26.

12. Chi C, Teliewubai J, Lu Y, Fan X, Yu S, Xiong J, et al. Comparison of various lipid parameters in association of target organ damage: a cohort study. Lipids Health Dis. 2018;17:198-9.

13. Tamada M, Makita S, Abiko A, Naganuma Y, Nagai M, Nakamura M. Lowdensity lipoprotein cholesterol to high-density lipoprotein cholesterol ratio as a useful marker for early-stage carotid atherosclerosis. Metabolism. 2010; 59:653-7.

14. Shah AS, Urbina EM, Khoury PR, Kimball TR, Dolan LM. Lipids and lipoprotein ratios: contribution to carotid intima media thickness in adolescents and young adults with type 2 diabetes mellitus. J Clin Lipidol. 2013;7:441-5.

15. Su $X$, Kong $Y$, Peng D. Evidence for changing lipid management strategy to focus on non-high density lipoprotein cholesterol. Lipids Health Dis. 2019; 18:134-7.

16. Joosten MM, Pai JK, Bertoia ML, Rimm EB, Spiegelman D, Mittleman MA, et al. Associations between conventional cardiovascular risk factors and risk of peripheral artery disease in men. JAMA. 2012;308:1660-7.

17. Levey AS, Stevens LA, Schmid CH, Zhang YL, Castro AF, Feldman HI, et al. A new equation to estimate glomerular filtration rate. Ann Intern Med. 2009; 150:604-12.

18. Alves-Cabratosa L, Garcia-Gil M, Comas-Cufi M, Blanch J, Ponjoan A, MartiLluch $\mathrm{R}$, et al. Role of low ankle-brachial index in cardiovascular and mortality risk compared with major risk conditions. J Clin Med. 2019;8:870.

19. Greenland P, Abrams J, Aurigemma GP, Bond MG, Clark LT, Criqui MH, et al. Prevention conference $\mathrm{V}$ : beyond secondary prevention: identifying the 
high-risk patient for primary prevention: · tests of atherosclerotic burden: writing group III. Circulation. 2000;101:E16-22.

20. Liang Y, Yan Z, Sun B, Cai C, Jiang H, Song A, et al. Cardiovascular risk factor profiles for peripheral artery disease and carotid atherosclerosis among Chinese older people: a population-based study. PLoS One. 2014;9:e85927.

21. Ridker PM, Stampfer MJ, Rifai N. Novel risk factors for systemic atherosclerosis: a comparison of C-reactive protein, fibrinogen, homocysteine, lipoprotein(a), and standard cholesterol screening as predictors of peripheral arterial disease. JAMA. 2001;285:2481-5.

22. Pradhan AD, Shrivastava S, Cook NR, Rifai N, Creager MA, Ridker PM. Symptomatic peripheral arterial disease in women: nontraditional biomarkers of elevated risk. Circulation. 2008;1 17:823-31.

23. Tongdee P, Loyd RA, Kanoksin S, Kanjanawetang J, Winwan K, Nimkuntod P. Clinical usefulness of lipid ratios to identify subclinical atherosclerosis in Perimenopausal/menopausal women. J Med Assoc Thail. 2016;99(Suppl 7): S36-41.

24. Zhan Y, Yu J, Ding R, Sun Y, Hu D. Triglyceride to high density lipoprotein cholesterol ratio, total cholesterol to high density lipoprotein cholesterol ratio and low ankle brachial index in an elderly population. Vasa. 2014;43: 189-97.

25. Jia L, Long S, Fu M, Yan B, Tian Y, Xu Y, et al. Relationship between total cholesterol/high-density lipoprotein cholesterol ratio, triglyceride/highdensity lipoprotein cholesterol ratio, and high-density lipoprotein subclasses. Metabolism. 2006;55:1141-8.

26. Song P, Rudan D, Wang M, Chang X, Rudan I. National and subnational estimation of the prevalence of peripheral artery disease (PAD) in China: a systematic review and meta-analysis. J Glob Health. 2019;9:010601.

27. Momin M, Jia J, Fan F, Li J, Dou J, Chen D, et al. Relationship between plasma homocysteine level and lipid profiles in a community-based Chinese population. Lipids Health Dis. 2017;16:54.

28. Yang N, Yao Z, Miao L, Liu J, Gao X, Xu Y, et al. Homocysteine diminishes apolipoprotein A-l function and expression in patients with hypothyroidism: a cross-sectional study. Lipids in health and disease. Lipids Health Dis. 2016; 15:123.

29. Aday AW, Lawler PR, Cook NR, Ridker PM, Mora S, Pradhan AD. Lipoprotein particle profiles, standard lipids, and peripheral artery disease incidence. Circulation. 2018;138:2330-41.

30. Mathews SC, Mallidi J, Kulkarni K, Toth PP, Jones SR. Achieving secondary prevention low-density lipoprotein particle concentration goals using lipoprotein cholesterol-based data. PLoS One. 2012;7:e33692.

31. Yokoyama K, Yokoyama K, Tani S, Tani S, Matsuo R, Matsuo R, et al. Increased triglyceride/high-density lipoprotein cholesterol ratio may be associated with reduction in the low-density lipoprotein particle size: assessment of atherosclerotic cardiovascular disease risk. Heart Vessel. 2019; 34:227-36.

32. Baigent C, Keech A, Kearney PM, Blackwell L, Buck G, et al. Pollicino C, efficacy and safety of cholesterol-lowering treatment: prospective metaanalysis of data from 90056 participants in 14 randomised trials of statins. Lancet. 2005:366:1267-78.

33. Milionis HJ, Rizos E, Kostapanos M, Filippatos TD, Gazi IF, Ganotakis ES, et al. Treating to target patients with primary hyperlipidaemia: comparison of the effects of ATOrvastatin and ROSuvastatin (the ATOROS study). Curr Med Res Opin. 2006:22:1123-31.

34. Sviridov D, Nestel P. Dynamics of reverse cholesterol transport: protection against atherosclerosis. Atherosclerosis. 2002:161:245-54.

35. Maeda S, Nakanishi S, Yoneda M, Awaya T, Yamane K, Hirano T, et al. Associations between small dense LDL, HDL subfractions (HDL2, HDL3) and risk of atherosclerosis in Japanese-Americans. J Atheroscler Thromb. 2012;19: 444-52.

36. Jeppesen J, Facchini FS, Reaven GM. Individuals with high total cholesterol/ HDL cholesterol ratios are insulin resistant. J Intern Med. 1998;243:293-8.

37. Salazar MR, Carbajal HA, Espeche WG, Leiva SC, Balbin E, Dulbecco CA, et al. Relation among the plasma triglyceride/high-density lipoprotein cholesterol concentration ratio, insulin resistance, and associated cardio-metabolic risk factors in men and women. Am J Cardiol. 2012;109:1749-53.

38. Chen J, Mohler ER, Xie D, Shlipak M, Townsend RR, Appel LJ, et al. Traditional and non-traditional risk factors for incident peripheral arterial disease among patients with chronic kidney disease. Nephrol Dial Transplant. 2016;31:1145-51.

39. Liao D, Tan H, Hui R, Li Z, Jiang X, Gaubatz J, et al. Hyperhomocysteinemia decreases circulating high-density lipoprotein by inhibiting apolipoprotein
A-l protein synthesis and enhancing HDL cholesterol clearance. Circ Res. 2006:99:598-606.

40. Seo H, Oh H, Park H, Park M, Jang Y, Lee M. Contribution of dietary intakes of antioxidants to homocysteine-induced low density lipoprotein (LDL) oxidation in atherosclerotic patients. Yonsei Med J. 2010;51:526.

41. Ferretti G, Bacchetti T, Moroni C, Vignini A, Nanetti L, Curatola G. Effect of homocysteinylation of low density lipoproteins on lipid peroxidation of human endothelial cells. J Cell Biochem. 2004;92:351-60.

\section{Publisher's Note}

Springer Nature remains neutral with regard to jurisdictional claims in published maps and institutional affiliations.
Ready to submit your research? Choose BMC and benefit from:

- fast, convenient online submission

- thorough peer review by experienced researchers in your field

- rapid publication on acceptance

- support for research data, including large and complex data types

- gold Open Access which fosters wider collaboration and increased citations

- maximum visibility for your research: over $100 \mathrm{M}$ website views per year

At BMC, research is always in progress.

Learn more biomedcentral.com/submissions 